\title{
A Kidney Transplant Recipient with Recurrent Henoch-Schönlein Purpura Nephritis Successfully Treated with Steroid Pulse Therapy and Epipharyngeal Abrasive Therapy
}

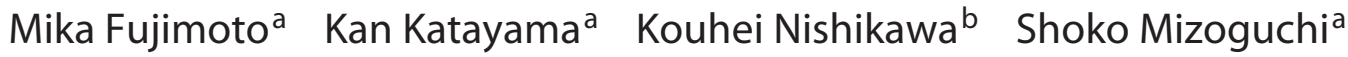 \\ Keiko Oda ${ }^{a}$ Yosuke Hirabayashi ${ }^{a}$ Yasuo Suzuki ${ }^{a}$ Ayumi Haruki ${ }^{a}$

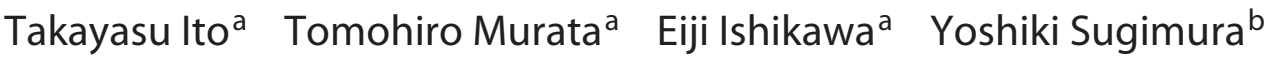 \\ Masaaki Ito ${ }^{a}$ \\ aDepartment of Cardiology and Nephrology, Mie University Graduate School of Medicine, Tsu, Japan; ${ }^{\text {bDepartment }}$ \\ of Nephro-Urologic Surgery and Andrology, Mie University Graduate School of Medicine, Tsu, Japan
}

\section{Keywords}

Epipharyngeal abrasive therapy $\cdot$ Henoch-Schönlein purpura nephritis · Kidney transplantation · Recurrent glomerulonephritis · Steroid pulse therapy

\begin{abstract}
There is no specific treatment for recurrent Henoch-Schönlein purpura nephritis (HSPN) in a transplanted kidney. We herein report a case of a kidney transplant recipient with recurrent HSPN that was successfully treated with steroid pulse therapy and epipharyngeal abrasive therapy (EAT). A 39-year-old Japanese man developed HSPN 4 years ago and had to start hemodialysis after 2 months despite receiving steroid pulse therapy followed by oral prednisolone, plasma exchange therapy, and cyclophosphamide pulse therapy. He had undergone tonsillectomy 3 years earlier in the hopes of achieving a better outcome of a planned kidney transplantation and received a living-donor kidney transplantation from his mother 1 year earlier. Although there were no abnormalities in the renal function or urinalysis 2 months after transplantation, a routine kidney allograft biopsy revealed evidence of mesangial proliferation and cellular cres-
\end{abstract}

cent formation. Mesangial deposition for $\lg \mathrm{A}$ and C3 was noted, and he was diagnosed with recurrent HSPN histologically. Since the renal function and urinalysis findings deteriorated 5 months after transplantation, 2 courses of steroid pulse therapy were performed but were ineffective. EAT using $0.5 \%$ zinc chloride solution once per day was combined with the third course of steroid pulse therapy, as there were signs of chronic epipharyngitis. His renal function recovered 3 months after daily EAT and has been stable for 1.5 years since transplantation. Daily EAT continued for $>3$ months might be a suitable strategy for treating recurrent HSPN in cases of kidney transplantation.

(c) 2020 S. Karger AG, Basel

\section{Introduction}

Henoch-Schönlein purpura nephritis (HSPN) is a clinical feature of Henoch-Schönlein purpura. HenochSchönlein purpura was described as IgA vasculitis with abnormal IgA deposits at the revised International Chapel Hill Consensus Conference in 2012 [1]. Therefore, the treatment of HSPN conforms to that of IgA nephropathy.

$\begin{aligned} & \text { karger@karger.com } \\ & \text { www.karger.com/nef }\end{aligned}$
Karger ${ }^{\prime /}$

Kan Katayama

Department of Cardiology and Nephrology

Mie University Graduate School of Medicine

2-174 Edobashi, Tsu 514-8507 (Japan)

katayamk@clin.medic.mie-u.ac.jp 
Fig. 1. Native kidney biopsy findings. a PAS stain. Global mesangial hypercellularity and segmental endocapillary proliferation with nuclear pyknosis were noted. The glomerular capillary walls were thickened with subendothelial deposits. b PAM stain showed crescent formation. IgA (c), C3 (d). A direct immunofluorescence study for IgA and C3 demonstrated numerous mesangial deposits as well as scattered deposits in the peripheral capillaries. Scale bars, $50 \mu \mathrm{m}$. PAS, periodic acid-Schiff; PAM, periodic acid methenamine silver.
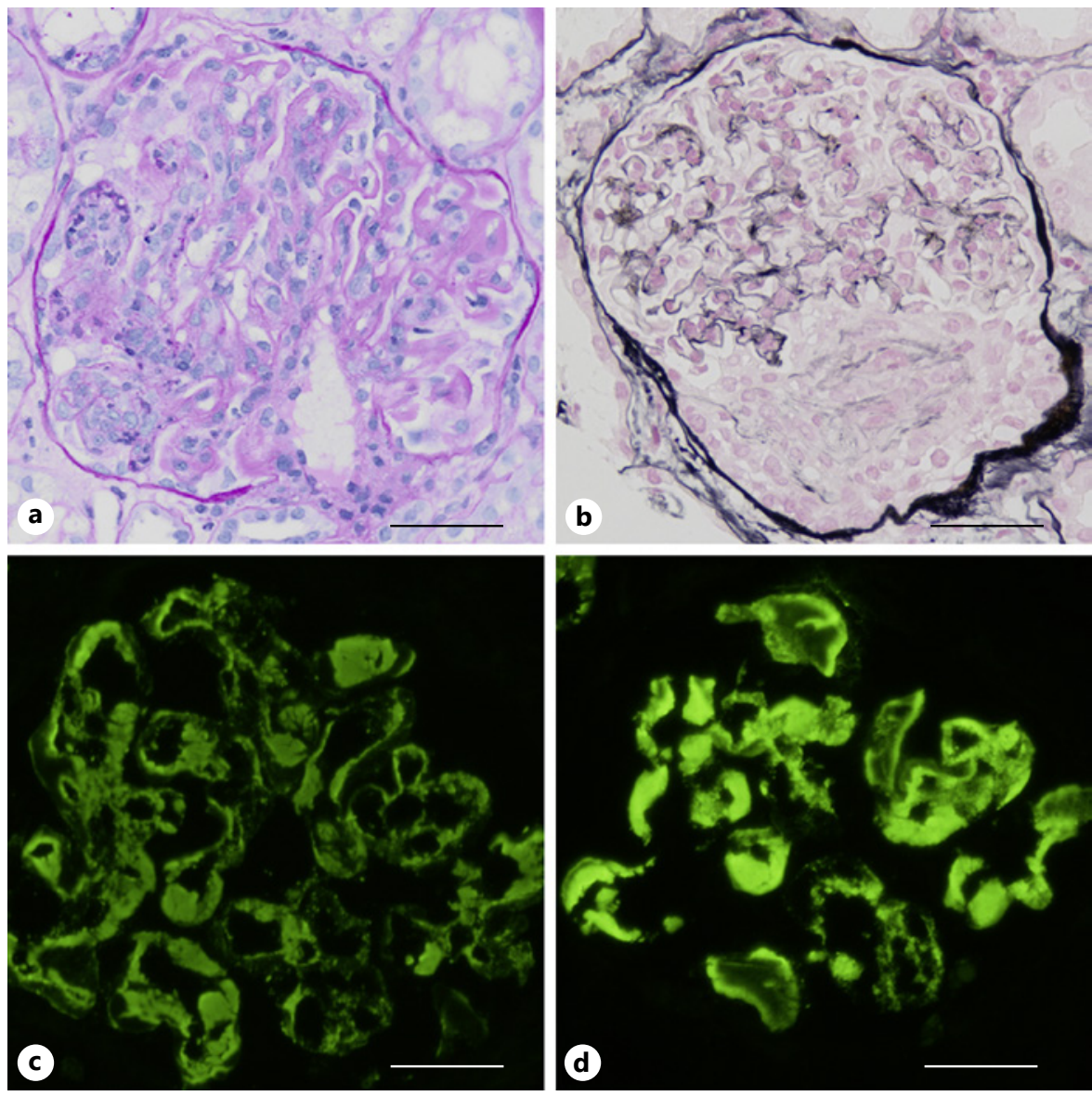

Some reports have described the efficacy of steroid pulse therapy, oral steroid therapy, tonsillectomy, immunosuppressive agents, and plasma exchange therapy in adult cases of HSPN [2-4]. However, controlled clinical trials for recurrent HSPN in patients with renal allografts are limited. Previous reports have shown the efficacy of steroid pulse therapy plus tonsillectomy or plasma exchange therapy in cases of recurrent HSPN in patients with renal allografts $[5,6]$. Although the association between chronic epipharyngitis and IgA nephropathy has been reported [7], this is the first report describing the efficacy of steroid pulse therapy plus epipharyngeal abrasive therapy (EAT) for recurrent HSPN in a patient with a renal allograft.

\section{Case Report/Case Presentation}

A 35-year-old Japanese man developed joint pain, edema, and purpura on his lower legs and visited our hospital. His serum $\mathrm{Cr}$ (S-Cr) level was $3.2 \mathrm{mg} / \mathrm{dL}$, and a urinalysis showed hematuria (urinary red blood cells [RBCs] 55-99/high-power field [HPF]) and $10 \mathrm{~g} / \mathrm{gCr}$ proteinuria. Antinuclear antibody, anti-neutrophil cytoplasmic antibodies, and cryoglobulin were all negative, and C3 and C4 levels were within normal ranges. A kidney biopsy showed endocapillary proliferative changes with pyknotic nuclear debris and cellular crescent formation in 6 of 9 glomeruli (shown in Fig. 1). Since an immunofluorescence study demonstrated $2+$ deposition of IgA and C3 in the mesangial and peripheral regions (shown in Fig. 1), he was diagnosed with HSPN grade Vb + VI according to the International Study of Kidney Disease in Children (ISKDC) classification. Extensive electron dense deposits were observed in the mesangial, paramesangial, and subendothelial regions on an electron microscopic study. As there was no response to 3 courses of steroid pulse therapy followed by oral prednisolone $(40 \mathrm{mg} /$ day), 5 courses of plasma exchange therapy were performed but were ineffective. Although cyclophosphamide pulse therapy was also performed, his renal function rapidly worsened, and he started to receive hemodialysis after 2 months.

Since his mother was willing to be a kidney donor, a kidney transplantation from his mother was planned. To achieve the best possible outcome of the kidney allograft, he expressed a desire to undergo tonsillectomy before transplantation, although he had initially refused to do so during treatments before hemodialysis. Therefore, he underwent tonsillectomy at 36 years of age and a living-donor kidney transplantation from his mother at 38 years of age. Although the $\mathrm{ABO}$ blood types were incom- 


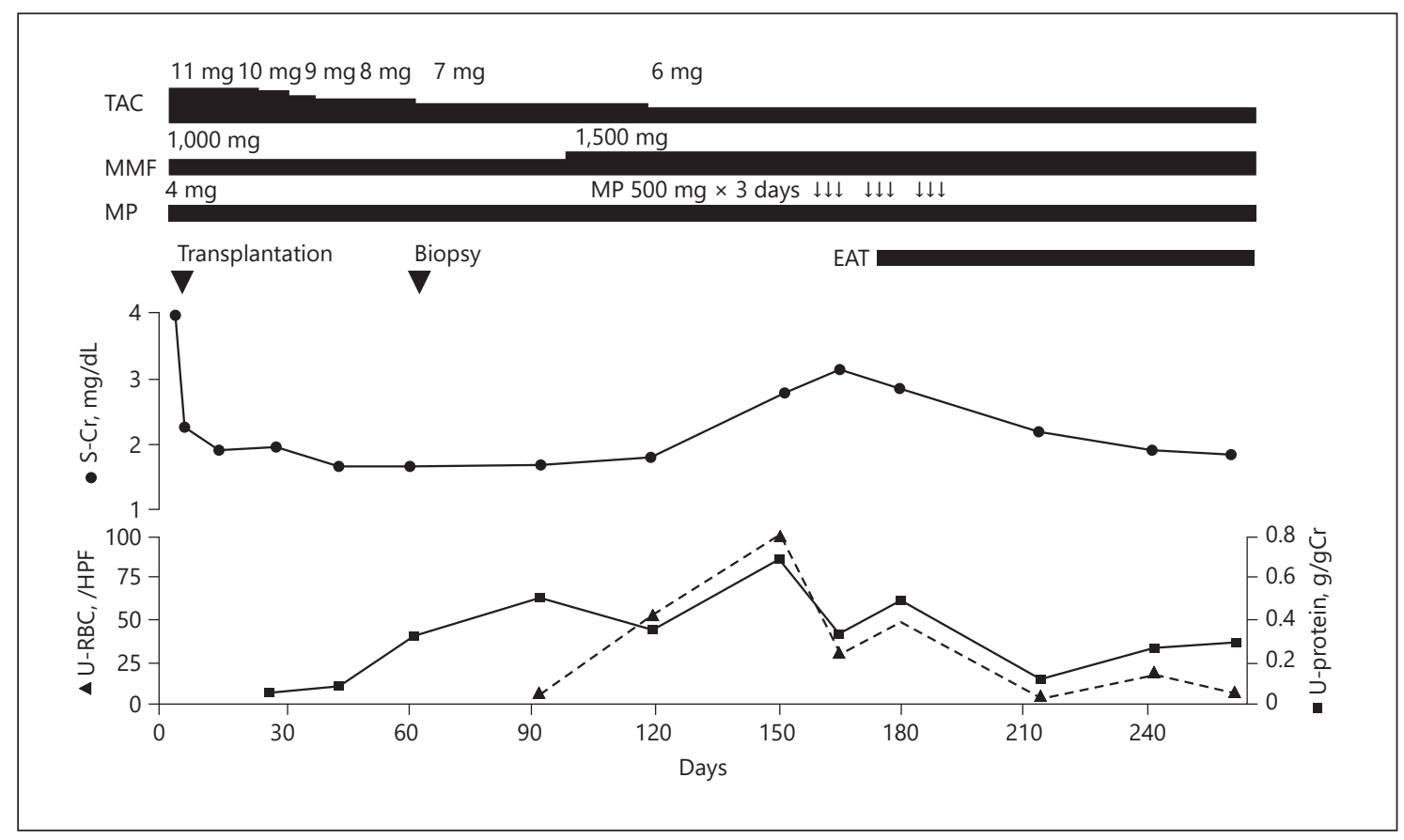

Fig. 2. Clinical course after kidney transplantation. S-Cr, U-protein, and U-RBC findings were improved after EAT therapy. S-Cr, serum Cr; U-protein, urinary protein; U-RBC, urinary red blood cells; EAT, epipharyngeal abrasive therapy; MMF, mycophenolate mofetil; MP, methylprednisolone; TAC, tacrolimus.

patible (B to A), anti-HLA antibody and anti-donor antibody were negative. Treatment was started with 5 immunosuppressive agents such as tacrolimus, mycophenolate mofetil (MMF), methylprednisolone, basiliximab, and rituximab (shown in Fig. 2). A baseline ( $0 \mathrm{~h}$ ) biopsy showed neither pre-existing disease nor IgA deposition. The S-Cr level was stable $(1.8-1.9 \mathrm{mg} /$ $\mathrm{dL})$ within 1 month after transplantation, and the urinalysis findings were normal with a good postoperative course.

There were no clinical symptoms for 2 months after transplantation. The S-Cr level was $1.7 \mathrm{mg} / \mathrm{dL}$, and a urinalysis showed mild proteinuria $(0.32 \mathrm{~g} / \mathrm{gCr})$ without hematuria. A routine kidney allograft biopsy showed no evidence of rejection (Banff classification; i0, t0, v0, g0, ptc0, c4d0, ci0, ct0, cv0 cg0, mm0, ah0, aah1); however, there were focal segmental mesangial matrix hypercellularity and endocapillary proliferative changes in 2 glomeruli, and 1 of 15 glomeruli showed a cellular crescent (shown in Fig. 3). There were no sclerosed glomeruli. An immunofluorescence study showed extensive depositions of $1+\operatorname{IgA}, 1+\mathrm{C} 3$ in mesangial areas (shown in Fig. 3). He was diagnosed with recurrent HSPN histologically. Microscopic hematuria emerged 3 months after transplantation, and MMF was increased from 1,000 mg/day to 1,500 $\mathrm{mg} /$ day. Since his renal function and urinalysis findings worsened (S-Cr $2.8 \mathrm{mg} / \mathrm{dL}$, urinary RBCs 30-49/HPF, and proteinuria $0.7 \mathrm{~g} /$ $\mathrm{gCr})$, he was treated with 3 courses of methylprednisolone pulse therapy ( $500 \mathrm{mg} /$ day for 3 days) every week.

His renal function was further deteriorated 6 months after the transplantation. S-Cr was $3.2 \mathrm{mg} / \mathrm{dL}$, and a urinalysis showed hematuria (urinary RBCs over 100/HPF) with RBC casts in the urine sediment. We suspected the involvement of chronic epi- pharyngitis in his progressive renal dysfunction because he had pharyngeal discomfort, sore throat, and chronic fatigue. A nasopharyngeal fiberscope revealed edema of the nasopharynx mucosa and easy bleeding by rubbing, which was compatible with the diagnosis of chronic epipharyngitis. EAT was started by inserting a swab with $0.5 \%$ zinc chloride $\left(\mathrm{ZnCl}_{2}\right)$ solution via the nasal cavity and rubbing for approximately $30 \mathrm{~s}$ once per day; this was performed at home by himself.

Three months after starting EAT therapy, his clinical symptoms, such as pharyngeal discomfort and sore throat, were ameliorated, and his renal function was improved (S-Cr level $1.9 \mathrm{mg} / \mathrm{dL}$ ), as was his proteinuria $(0.3 \mathrm{~g} / \mathrm{gCr})$ and hematuria (urinary $\mathrm{RBCs}$ 5-9/HPF). Ten months after the transplantation, a kidney biopsy was performed to determine the therapeutic effect. Although mesangial cell proliferation and endocapillary changes remained, crescent formation was not observed. The depositions of IgA and $\mathrm{C} 3$ in the mesangial regions remained. Although microscopic hematuria also remained, there was no proteinuria, and his S-Cr level was stable at about $2.0 \mathrm{mg} / \mathrm{dL} 1$ and a half years after transplantation (shown in Fig. 2). There were no serious complications, such as infection or osteoporosis.

\section{Discussion/Conclusion}

We experienced a case of recurrent HSPN in a patient with a renal allograft that was successfully treated with steroid pulse therapy and EAT. While the recurrence 
Fig. 3. Routine allograft biopsy findings 2 months after transplantation. PAS stain (a), PAM stain (b). Mesangial hypercellularity and matrix expansion with a cellular crescent formation were noted. IgA (c), C3 (d). A direct immunofluorescence study showed mild deposition of IgA and C3 in the mesangial regions. Scale bars, $50 \mu \mathrm{m}$. PAS, periodic acid-Schiff; PAM, periodic acid methenamine silver.
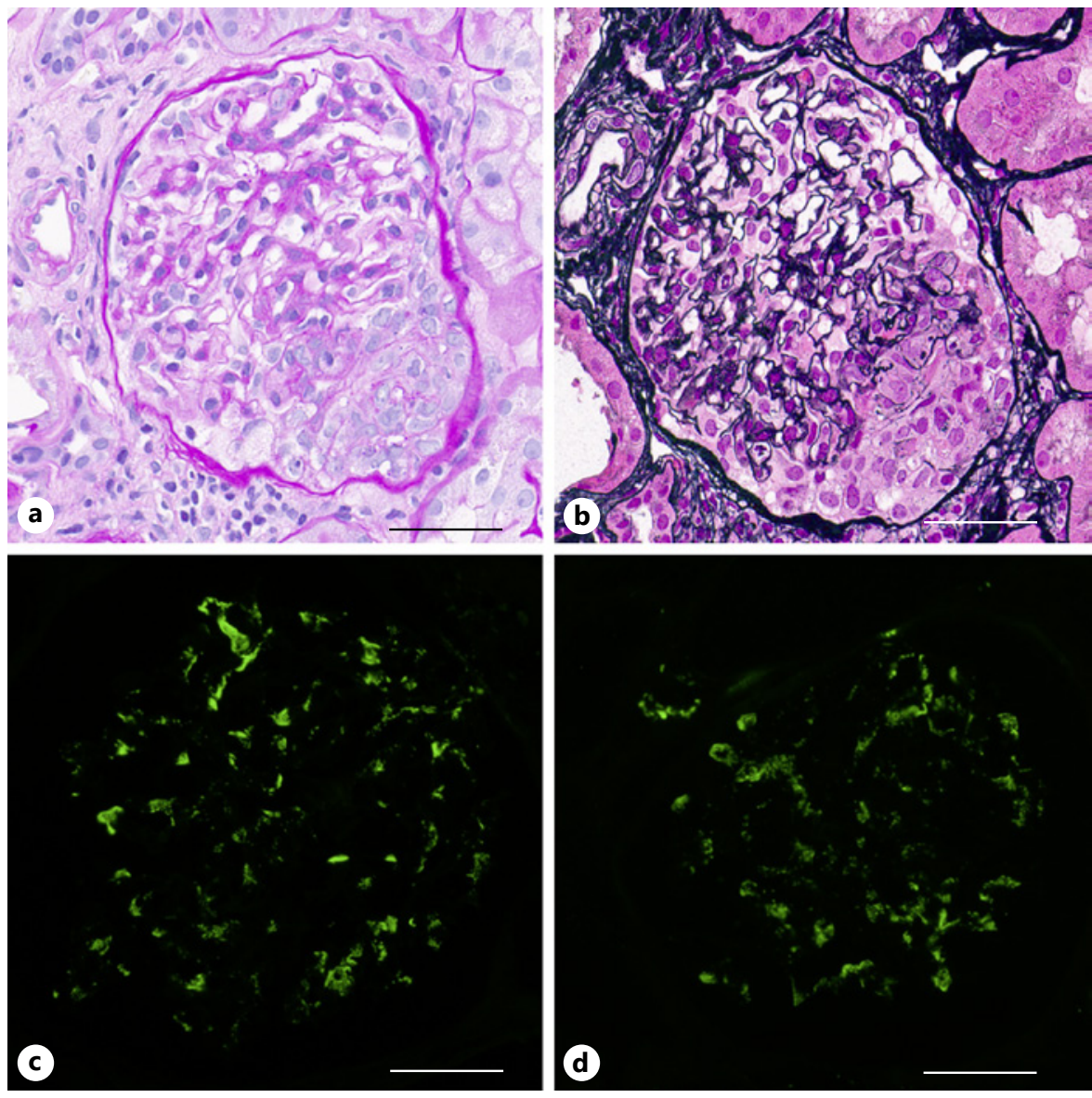

rates of HSPN and graft loss reported in 1994 were 35 and $11 \%$, respectively [8], at 5 years post-transplantation, these rates had improved to 11.5 and $7.5 \%$ at 10 years post-transplantation in 2011 [9]. However, graft loss from recurrence occurred in $13.6 \%$ of cases of HSPN but in $6.6 \%$ of cases of non-HSPN [10]. It was also reported that $71 \%$ of HSPN patients with crescentic glomerulonephritis in the native kidneys developed recurrence of HSPN [11]. Thus, it is important to control recurrent HSPN in patients with renal allografts.

The risk of recurrence of HSPN was thought to be high in the present case because crescent formation was observed in 6 of 9 glomeruli in the native kidney. Tonsillectomy was performed 2 years before kidney transplantation. Nevertheless, the histological recurrence of HSPN had already been observed 2 months after transplantation. A previous report suggested that tonsillectomy before kidney transplantation had no beneficial effect on the outcome of kidney allograft in IgA nephropathy [12]. The clinical recurrence of HSPN without extra-renal symptoms was observed at 3 months after transplantation and was also resistant to steroid pulse therapy alone. We therefore considered the potential existence of an association between chronic epipharyngitis and recurrence of purpura nephritis.

The clinical symptoms that were observed in $>10 \%$ of patients with chronic epipharyngitis include headache, postnasal drip, pharyngeal discomfort, chronic cough, neck \& shoulder stiffness, sore throat, dizziness, chronic fatigue, and irritable bowel syndrome [7]. Chronic epipharyngitis is characterized by prominent nasal submucosal edema on laryngoscopy, and EAT is reported to be effective for its diagnosis and treatment [7]. EAT is typically performed by rubbing the epipharyngeal area with a cotton laryngeal swab soaked with $0.5 \% \mathrm{ZnCl}_{2}$ solution daily to improve venous and lymphatic stasis [7]. There was a report that the addition of EAT to steroid pulse therapy and mizoribine was effective for improving IgA nephropathy in the native kidney [13]. Since he did not have any extra-renal manifestations, the depositions of IgA and C3 2 months after transplantation could thus have also been diagnosed as 
IgA nephropathy. If the present case was indeed one of IgA nephropathy, its MEST-C scores would be M0, E1, S0, T0, C1.

The putative mechanisms underlying EAT are considered to be the anti-inflammatory effect of $\mathrm{ZnCl}_{2}$, the blood-letting effect of EAT, and vagus nerve stimulation by EAT [7]. Vagus nerve stimulation has also been reported to be effective in a kidney ischemia-reperfusion injury mouse model [14]. To our knowledge, this is the first case in which EAT was effective in a patient with a renal allograft who had recurrent HSPN. The concomitant application of the third course of steroid pulse therapy and EAT might have been responsible for the beneficial results in the present case. EAT may be a suitable option for treating recurrent HSPN when there are signs of chronic epipharyngitis. EAT needs to be performed with proper supervision in order to avoid reducing olfaction. Daily EAT continued for more than 3 months was effective for stopping the progression of renal dysfunction in the present case. EAT might therefore be a suitable strategy for treating recurrent HSPN in cases of kidney transplantation.

\section{Statement of Ethics}

Written informed consent was obtained from the patient for the publication of this case report.

\section{Conflict of Interest Statement}

The authors have no conflicts of interest to declare.

\section{Funding Sources}

Not applicable.

\section{Author Contributions}

M.F., K.K., K.N., T.M. analyzed the clinical course. S.M., K.O., Y.H., Ya.S., A.H., T.I. evaluated the pathology finding of the kidney biopsies. M.F. and K.K. wrote a draft of the manuscript, and K.N., S.M., K.O., Y.H., Ya.S., A.H., T.I., T.M., E.I., Yo.S., M.I. revised it critically. All authors read and approved the final manuscript.

\section{References}

1 Jennette JC, Falk RJ, Bacon PA, Basu N, Cid MC, Ferrario F, et al. 2012 revised international chapel hill consensus conference nomenclature of vasculitides. Arthritis Rheum. 2013;65(1):1-11.

2 Sugiyama $\mathrm{H}$, Watanabe $\mathrm{N}$, Onoda T, Kikumoto Y, Yamamoto M, Maeta M, et al. Successful treatment of progressive HenochSchönlein purpura nephritis with tonsillectomy and steroid pulse therapy. Intern Med. 2005;44(6):611-5.

3 Sasaki E, Shibata M, Kato A, Hamano N, Katsuki $T$, Tada $M$, et al. An adult case of severe steroid-resistant Henoch-Schönlein purpura nephritis treated with intravenous cyclophosphamide and tonsillectomy. CEN Case Rep. 2016;5(2):212-8.

4 Kauffmann RH, Houwert DA. Plasmapheresis in rapidly progressive Henoch-Schoenlein glomerulonephritis and the effect on circulating IgA immune complexes. Clin Nephrol. 1981;16(3):155-60.

5 Yamakawa T, Yamamoto I, Komatsuzaki Y, Niikura T, Okabayashi Y, Katsumata H, et al. Successful treatment of recurrent Henoch-
Schönlein purpura nephritis in a renal allograft with tonsillectomy and steroid pulse therapy. Nephrology. 2016;21(Suppl 1):53-6.

6 Lee J, Clayton F, Shihab F, Goldfarb-Rumyantzev A. Successful treatment of recurrent Henoch-Schönlein purpura in a renal allograft with plasmapheresis. Am J Transplant. 2008;8(1):228-31.

7 Hotta O, Inoue CN, Tanaka A, Ieiri N. Possible mechanisms underlying epipharyngeal abrasive therapy (EAT) with $\mathrm{ZnCl} 2$ solution for the treatment of autoimmune diseases and functional somatic syndrome. J Antivir Antiretrovir. 2017;9(4):168.

8 Meulders Q, Pirson Y, Cosyns JP, Squifflet JP, van Ypersele de Strihou C. Course of HenochSchönlein nephritis after renal transplantation. Report on ten patients and review of the literature. Transplantation. 1994;58(11):1179-86.

9 Kanaan N, Mourad G, Thervet E, Peeters P, Hourmant M, Vanrenterghem Y, et al. Recurrence and graft loss after kidney transplantation for henoch-schonlein purpura nephritis: a multicenter analysis. Clin J Am Soc Nephrol. 2011;6(7):1768-72.
10 Samuel JP, Bell CS, Molony DA, Braun MC Long-term outcome of renal transplantation patients with Henoch-Schonlein purpura. Clin J Am Soc Nephrol. 2011;6(8):2034-40.

11 Moroni G, Gallelli B, Diana A, Carminati A, Banfi G, Poli F, et al. Renal transplantation in adults with Henoch-Schonlein purpura: longterm outcome. Nephrol Dial Transplant. 2008;23(9):3010-6.

12 Sato Y, Ishida H, Shimizu T, Tanabe K. Evaluation of tonsillectomy before kidney transplantation in patients with IgA nephropathy. Transpl Immunol. 2014;30(1):12-7.

13 Kaneko T, Mii A, Fukui M, Nagahama K, Shimizu A, Tsuruoka S. IgA nephropathy and psoriatic arthritis that improved with steroid pulse therapy and mizoribine in combination with treatment for chronic tonsillitis and epipharyngitis. Intern Med. 2015;54(9):108590.

14 Inoue T, Abe C, Sung SS, Moscalu S, Jankowski J, Huang L, et al. Vagus nerve stimulation mediates protection from kidney ischemiareperfusion injury through $a 7 \mathrm{nAChR}+$ splenocytes. J Clin Invest. 2016;126(5):1939-52. 